\title{
Nicotine Exposure Augments Renal Toxicity of 5-aza- cytidine Through p66shc: Prevention by Resveratrol
}

\author{
ISTVAN ARANY $^{1}$, SAMUEL HALL $^{1}$, AMIR FAISAL $^{2}$ and MEHUL DIXIT ${ }^{1}$ \\ ${ }^{1}$ Department of Pediatrics, Division of Pediatric Nephrology, \\ University of Mississippi Medical Center, Jackson, MS, U.S.A.; \\ ${ }^{2}$ Lahore University of Management Sciences, Lahore, Pakistan
}

\begin{abstract}
Background/Aim: We have shown that either chronic nicotine (NIC) exposure or 5-aza-cytidine (AZA) augments oxidative stress-dependent injury through stimulating p66shc in renal cells. Hence, NIC could exacerbate adverse effects of AZA while antioxidants such as resveratrol (RES) could prevent it. Materials and Methods: Renal proximal tubule cells (NRK52E) were treated with $20 \mu \mathrm{M}$ RES prior to $200 \mu \mathrm{M}$ NIC plus $100 \mathrm{nM}$ AZA and cell injury (LDH release) was determined. Reporter luciferase assays determined p66shc activation and RES-induced antioxidant responses. Genetic manipulations identified the mechanism of RES action. Results: NIC exacerbated AZAdependent injury via augmenting p66shc transcription. While RES suppressed NIC+AZA-mediated injury, -surprisingly- it further enhanced activity of the p66shc promoter. RES protected cells via the cytoplasmic p66shc/Nrf2/heme oxygenase-1 (HO1) axis. Conclusion: RES can protect the kidney from adverse effects of NIC in patients undergoing anticancer therapy.
\end{abstract}

Studies demonstrated that nephrotoxicity is a frequent side-effect of various anticancer agents that hampers their clinical use (1). Previously we reported that the anticancer agent 5-aza-cytidine (AZA) elicits oxidative stress-mediated toxicity in cultured renal proximal tubule cells via transcriptional upregulation of the prooxidant p66shc gene and consequent increase in mitochondrial reactive oxygen species (ROS) release (2). Studies revealed that smoking augments severity and progression of renal diseases in humans (3) and in various animal models (4-6). However, there are no data available on whether smoking could affect renal toxicity of anticancer agents.

This article is freely accessible online.

Correspondence to: Istvan Arany, Department of Pediatrics, Division of Pediatric Nephrology, University of Mississippi Medical Center, Jackson, MS, U.S.A. Tel: +1 6018159464, Fax: +1 6019845981,e-mail: iarany@umc.edu

Key Words: 5-aza-cytidine, nicotine, renal-toxicity, p66shc, resveratrol
Smoking exerts its adverse effects via nicotine (NIC)induced oxidative stress (7). We reported that NIC transcriptionally activates and Serine 36 phosphorylates p66shc that results in its mitochondrial translocation and therein cytochrome $c$ binding and consequent excess ROS production (8) similar to AZA (2). Hence, it is plausible that NIC exposure exacerbates renal toxicity of AZA by further augmenting p66shc expression and resultant ROS production.

Resveratrol (RES) is a powerful dietary antioxidant (9) that has been shown to protect the kidney from cigarette smoke-associated injury in rats (10). RES exerts antioxidant effect - at least partly - through activation of the Nrf2/HO1 axis $(11,12)$. Whether beneficial effect of RES on NIC+AZA-exposed renal cells are due to activation of Nrf2/HO-1 and/or down-regulation of p66shc is unknown.

Accordingly, we hypothesized that (i) NIC exposure additively augments AZA-dependent induction of p66shc transcription, thus, exacerbates AZA-associated injury and (ii) RES attenuates NIC+AZA-mediated injury by suppressing p66shc transcription and/or activation of HO-1 in cultured renal proximal tubule cells.

\section{Materials and Methods}

Cell culture and treatment. The rat proximal tubule cell line (NRK52E) was maintained in $5 \% \mathrm{CO}_{2}$ at $37^{\circ} \mathrm{C}$ in DMEM with $10 \%$ FBS as recommended. A set of cultures was treated with $200 \mu \mathrm{M}$ nicotine (NIC; Sigma-Aldrich, St. Louis, MO, USA) or $100 \mathrm{nM} 5-$ aza-cytidine (AZA; Sigma-Aldrich) as described earlier in mouse proximal tubule cells (2). Some cultures were co-treated with NIC+AZA. Resveratrol (RES; Selleckchem, Houston, TX, USA) was applied in $20 \mu \mathrm{M}$ concentration overnight prior to NIC+AZA treatment.

Determination of cell injury. Extent of cell injury was determined by LDH release using the fluorescent "Cytotox-One Homogeneous Membrane Integrity" kit (Promega, Madison, WI) as described earlier (13). Briefly, cells grown in 96-well-plates were transfected and treated as described in the appropriate section and $24 \mathrm{~h}$ later $\mathrm{LDH}$ content of the supernatants were determined and compared to total 
LDH content of untreated cell lysate. Values were calculated as $\%$ of total LDH content and expressed as \% of control (untreated) values.

Reporter luciferase assay. NRK52E cells were transfected with the following reporter luciferase plasmids: p66shc promoter (14), antioxidant response element (ARE; Qiagen, Germantown, MD USA) and a HO-1-promoter (15) together with a Renilla luciferase (Promega, Madison, WI, USA) using Lipofectamine 3000 reagent (Life Technologies, Grand Island, NY, USA) as recommended by the manufacturer. $24 \mathrm{~h}$ after treatment firefly and renilla luciferase activities were determined using the Dual Luciferase assay kit (Promega, Madison, WI, USA) as recommended by the manufacturer. Luciferase activities were calculated as ratios of the firefly and renilla activities and expressed as a percentage of the control (untreated) values.

Genetic manipulation of cellular targets. To knockdown p66shc, NRK52E cells were transfected with a short-hairpin-p66shc (shp66shc) plasmid (16). To prevent Serine36 phosphorylation or mitochondrial cytochrome $c$ binding of p66shc, cells were transfected with a Serine-36 phosphorylation-deficient mutant p66shc (S36A) or a cytochrome c-binding-deficient mutant p66shc (W134F) as described elsewhere (16). Nrf2 expression was knocked down by a Nrf2 siRNA (Santa Cruz, Ja Jolla, CA) using Lipofectamine 3000 (Life Technologies, Grand Island, NY, USA) as previously described (17). HO-1 expression was knocked down by an HO-1 siRNA (Santa Cruz, La Jolla, CA) using Lipofectamine 3000 (Life Technologies, Grand Island, NY, USA).

Statistical analysis. Continuous variables are expressed as means and standard deviations (S.D.). One-way ANOVA with Holm-Sidak post-hoc test was used to evaluate differences between groups. Differences between means were considered significant if $p<0.05$. All analyses were performed using the GraphPad InStat3 (La Jolla, CA, USA) software package.

\section{Results}

Nicotine treatment augments AZA-dependent cytotoxicity via p66shc and related oxidative stress in renal proximal tubule cells. NRK52E cells were treated with either $200 \mu \mathrm{M}$ NIC or $100 \mathrm{nM}$ AZA or with their combination and LDH release was determined $24 \mathrm{~h}$ later. Figure 1A shows that both AZA and NIC increased LDH release similar to that found in a mouse proximal tubule cell line $(8,18)$. Importantly, combination of NIC and AZA was additive. Previously we demonstrated that both NIC (8) and AZA (2) exerts cytotoxicity via p66shc. Hence, it is not surprising that knockdown of p66shc (shp66shc) attenuated NIC+AZA-associated increase in LDH release (Figure 1A). Since p66shc exerts its cytotoxic effect via increasing oxidative stress $(2,8)$, we determined whether $\mathrm{NIC}+\mathrm{AZA}$ will do the same. Accordingly, cells were pretreated with the antioxidant $\mathrm{N}$-acetyl-cysteine (NAC; $100 \mu \mathrm{M}$ ) and NIC+AZA-mediated LDH release was determined. Figure 1A shows that NAC attenuated NIC+AZA-mediated injury. In addition, activity of the p66shc promoter was determined in a reporter luciferase assay, which revealed that NIC+AZA additively induces the p66shc promoter (Figure 1B).
Resveratrol inhibits NIC $+A Z A$-mediated $L D H$ release while augments p66shc transcription. NRK52E cells were pretreated with $20 \mu \mathrm{M}$ RES overnight prior to treatment with $200 \mu \mathrm{M}$ NIC+100 nM AZA and LDH release was determined. Figure $1 \mathrm{~A}$ shows that RES pretreatment almost completely abolished NIC+AZA-mediated cytotoxicity. Since $\mathrm{NIC}+\mathrm{AZA}$-dependent cytotoxicity is mediated through p66shc (Figure 1) we tested whether RES inhibits NIC+AZAmediated induction of the p66shc promoter. Accordingly, NRK52E cells were transfected with a p66shc promoter luciferase plasmid and pretreated with $20 \mu \mathrm{M}$ RES followed by treatment with $200 \mu \mathrm{M} \mathrm{NIC+100} \mathrm{nM} \mathrm{AZA,} \mathrm{as} \mathrm{described.}$ Figure 1B shows that -surprisingly- RES did not inhibit, but rather augmented NIC+AZA-dependent activation of the p66shc promoter, even though LDH release was inhibited under this condition (Figure 1A). In fact, RES itself modestly but significantly activated the p66shc promoter (Figure 1B) without increasing cellular toxicity (Figure 1A).

Resveratrol activates the antioxidant response element (ARE) via pSer36p66shc and Nrf2. To test whether RES induces antioxidant responses, activity of the antioxidant response element (ARE) was tested in NRK52E cells $24 \mathrm{~h}$ after treatment with $20 \mu \mathrm{M}$ RES. Figure $2 \mathrm{~A}$ shows that RES significantly activated the ARE reporter. We also showed that knockdown of Nrf2 (siNrf2) -a transcription factor that binds and hence, activates the ARE (19)- inhibited RES-dependent induction of the ARE (Figure 2A). Interestingly, inhibition of Ser36 phosphorylation of p66shc (S36A) also attenuates RES-dependent activation of the ARE (Figure 2A) while inhibition of mitochondrial cytochrome $c$ binding of p66shc (W134F) does not.

Resveratrol induces the HO-1 promoter via pSer36p66shc and Nrf2. The activated Nrf2 -via the ARE- induces the HO-1 promoter (15). Accordingly, NRK52 cells were transfected with a HO-1 promoter luciferase reporter together with a renilla luciferase as described in Materials and Methods. Figure 2B shows that RES treatment significantly induced it, the extent of which is attenuated by knockdown of Nrf2 (siNrf2) or inhibition of Ser36 phosphorylation of p66shc (S36A) but not by inhibition of its mitochondrial cytochrome $c$ binding (W134F).

Protective effect of resveratrol requires pSer36p66shc, Nrf2 and $H O-1$. In the next set of experiments, we determined whether RES mediates its protective effects via the pSer36p66shc/ Nrf2/HO-1 pathway. Figure 3A shows that knockdown of HO1 (siHO-1) or Nrf2 (siNrf2) completely abolished protective effects of RES on NIC+AZA-mediated cytotoxicity. In addition, inhibition of Ser36 phosphorylation of p66shc (S36A) but not its (mitochondrial) cytochrome $c$ binding (W134) also significantly attenuated beneficial effects of RES. 

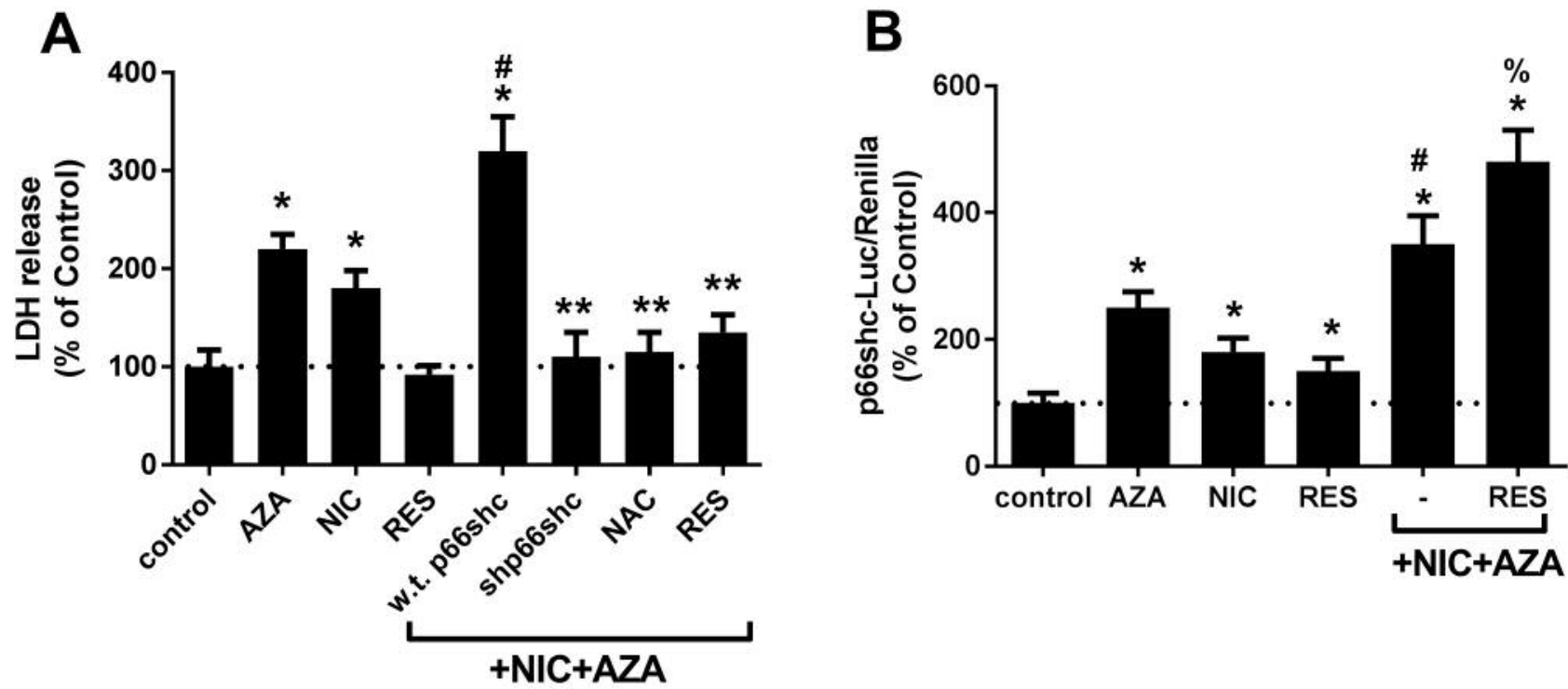

Figure 1. Nicotine exposure exacerbates toxicity of AZA via p66shc-dependent oxidative stress, which is alleviated with resveratrol. (A) NRK52E cells were treated with either $100 \mathrm{nM}$ AZA or $200 \mu \mathrm{M}$ NIC or with their combination and LDH release was determined 24 h later. In some experiments p66shc was knocked-down by transfection of a short-hairpin p66shc construct (shp66shc). Oxidative stress was prevented by pre-treatment with $100 \mu \mathrm{M} N A C$. A set of cells were pretreated with $20 \mu M$ RES overnight prior to treatment with NIC+AZA. N=3; ${ }^{*}<<0.05$ compared to control (untreated) cells; ${ }^{\#}<<0.05$ compared to either AZA or NIC alone; **p<0.05 compared to NIC $+A Z A$ in wild-type p66shc (w.t.p66shc) cells. (B) NRK52E cells were co-transfected

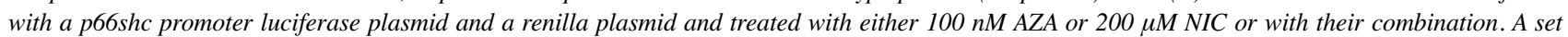
of cells were pretreated with $20 \mu M$ RES overnight prior to treatment with NIC+AZA. 24 h later luciferase activities were determined. Values were calculated as firefly (p66shc) and renilla ratios and expressed as \% of control values. $N=3 ;{ }^{*} p<0.05$ compared to control (untreated) cells; ${ }^{*} p<0.05$ compared to either AZA or NIC; $\% p<0.05$ compared to NIC $+A Z A$ values.

\section{Discussion}

Studies have suggested that smoking -primarily via NICworsens prognosis in cancer patients by interfering with cancer therapies (20) and potentially increasing their toxicity (21). However, effect of smoking on renal toxicity of anticancer agents has not been thoroughly evaluated. Several studies -including ours- have shown that NIC exposure/smoking exacerbates severity of acute (4) and chronic (6) kidney injury in experimental models and in the renal patient (3). Therefore, it is highly plausible that smoking/NIC exposure can enhance renal toxicity of anticancer agents. Previously we reported that the anticancer agent AZA increases oxidative stress-related injury in renal proximal tubule cells $(2,18)$. It is that AZA transcriptionally activates the p66shc promoter and phosphorylates Ser36 residue of the p66shc protein, which facilitates its mitochondrial translocation and binding to mitochondrial cytochrome $c$ (2). The result is enhanced mitochondrial ROS production and consequent mitochondrial depolarizationdependent injury (2). We revealed similar mechanism of injury by NIC treatment in renal proximal tubule cells (8). In this study, we determined that AZA-dependent toxicity is exacerbated by NIC, which is p66shc- and oxidative stress- dependent (Figure 1A). Based on our previous studies it can be substantiated that this oxidative stress is in fact, due to activation of p66shc. We also proved that adverse effect of NIC is due to augmented transcription of the p66shc gene (Figure 1B). Further studies are needed to determine the mechanism of this activation, which could involve p53 and epigenetic changes in the p66shc promoter $(2,8)$.

RES is a powerful antioxidant, protective effects of which is -at least partly- due to Nrf2-dependent induction of HO1 (22). Indeed, RES activates the ARE via Nrf2 (Figure 2A), which induces the HO-1 promoter (Figure 2B) that leads to protection against NIC+AZA-mediated cytotoxicity (Figure $3 \mathrm{~A}$ ) in renal proximal tubule cells. However, besides Nrf2, p66shc also participates in RES-afforded cytoprotection (Figure 3A) even though p66shc also plays a role in NIC+AZA-mediated cytotoxicity (Figure 1A).

p66sch is known as a pro-oxidant enzyme that links oxidative stress to renal injury $(2,8,23,24)$. Interestingly, lately some studies -including ours (17)- revealed that under certain circumstances p66shc can serve as an antioxidant (25). It is that the Ser36 phsophorylated p66shc does not translocate to the mitochondria - as during its prooxidant function - but rather activates the ARE and hence, a set of antioxidant genes that harbors the ARE in their 


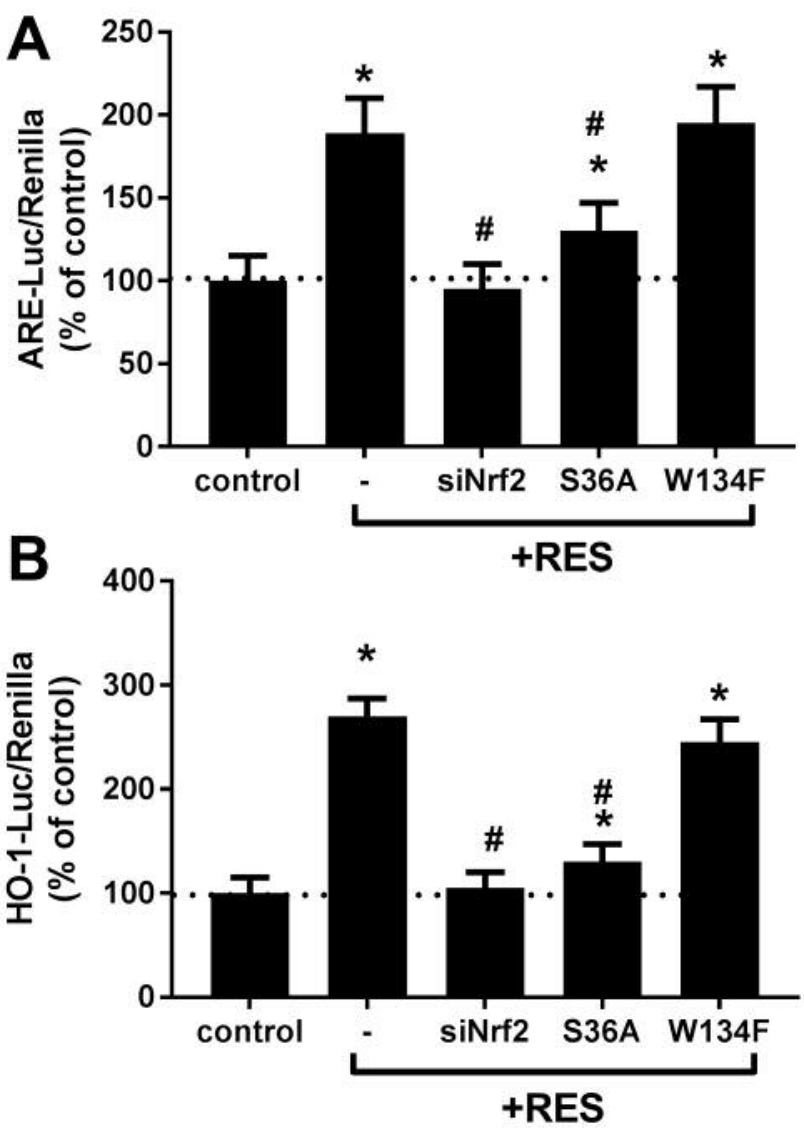

Figure 2. Resveratrol activates the antioxidant response element (ARE) and the HO-1 promoter via Nrf2 and pSer36p66shc. (A) NRK52 cells were co-transfected with and ARE luciferase and renilla plasmid and treated with $20 \mu M$ RES. 24 h later luciferase activities were determined. Some sets were also co-transfected with either a Nrf2 siRNA (siNrf2) to knockdown Nrf2, S36Ap66shc (S36A) to inhibit Ser36 phosphorylation or W134Fp66shc (W134F) to inhibit mitochondrial cytochrome c binding of p66shc. Values were calculated and expressed as in Figure 2B. $N=3$; ${ }^{*} p<0.05$ compared to (untreated) control. ${ }^{\#} p<0.05$ compared to RES treatment. Dotted line represents control value. (B) NRK52E cells were co-transfected with an $\mathrm{HO}-1$ promoter luciferase and renilla plasmid and treated with $20 \mu M$ RES. Some sets were also co-transfected with either a Nrf2 siRNA (siNrf2), S36Ap66shc (S36A) or W134Fp66shc (W134F) plasmid as described in (A). Vales were calculated and expressed as described above. $N=3 ; * p<0.05$ compared to (untreated) control. ${ }^{*} p<0.05$ compared to RES treatment. Dotted line represents control value.

promoter. This phenomenon has been described in erythroleukemia cells upon hemin treatment (25) and by us in renal proximal tubule cells upon coenzyme Q10 treatment (17). Some studies demonstrated that RES protects keratinocytes (26) and prostate cells (27) from injury via activating (Ser36 phosphorylating) p66shc. However, the mechanism of this protective effect has not been elucidated. We believe that those results with RES suggest a similar mechanism to that described with hemin and coenzyme
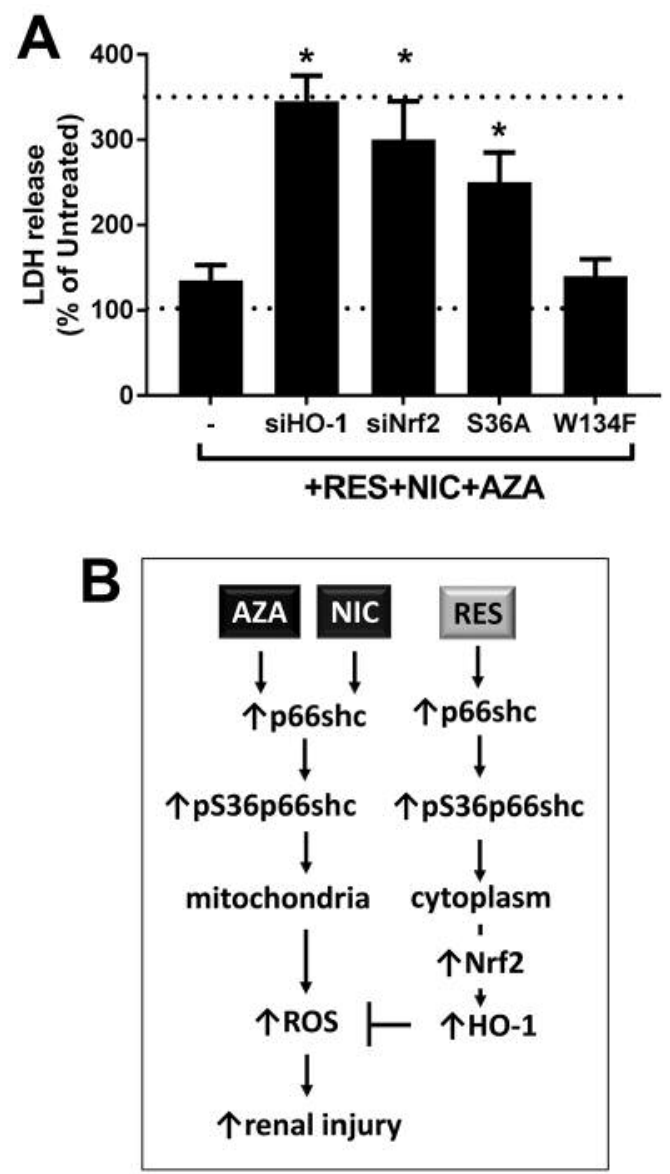

Figure 3. Protection by resveratol is afforded by pS36p66shc mediated activation of Nrf2 and consequent HO-1 induction. (A) NRK52 cells were transfected with either an HO-1 siRNA (siHO-1), Nrf2 siRNA (siNrf2), Ser36 (S36A)- and mitochondrial cytochrome c binding-deficient (W134F) p66shc plasmid and treated with RES+NIC+AZA as described above. 24 $h$ later $L D H$ release was determined. $N=3 ; * p<0.05$ compared to control. Lower dotted line represents control (untreated) value. Upper dotted line depicts NIC+AZA-treatment value. (B) Summary of our findings. NIC +AZA augments p66shc transcription and its Ser36 phosphorylation that results in mitochondrial translocation and -via binding to cytochrome $c-R O S$ release, which injures the cells. RES treatment also augments p66shc transcription and its Ser36 phosphorylation, which is retained in the cytosol and activates the $\mathrm{HO}-1$ promoter via $\mathrm{Nrf}$. The activated $\mathrm{HO}$ 1 exerts antioxidant/protective effects.
Q10. Here, we showd that RES protects renal proximal tubule cells against NIC+AZA-mediated oxidative stress (Figure 1A), which requires Ser36 phosphorylation (but not mitochondrial localization) of p66shc (Figure 3A). While RES Ser36 phosphorylates p66shc (Figure 2) it does not translocate and bind to mitochondrial cytochrome $c$ (see S36A and W134F data): instead, it activates the ARE and HO-1 promoter (Figure 2) resulting in cytoprotection (Figure 3). 
Our results rationalize that renal toxicity of AZA is more severe in smokers than in non-smokers. It is highly plausible that renotoxicity-enhancing effects of smoking might be applicable to other anticancer agents that activate p66shc. Previously we described that besides AZA, trichostatin A (2), taxol and dichloro-acetic acid (28) also exert their renal toxicity via activation of p66shc. While the best prevention would be smoking cessation, some long-term smokers refuse or can't quit. Hence, appropriate therapies might be needed. Our data offer therapeutic means to alleviate adverse effects of smoking on renotoxic effects of cancer therapies.

\section{Acknowledgements}

This work was supported by a grant from the Department of Pediatrics at the University of Mississippi Medical Center and by the Bower Foundation.

\section{References}

1 Skinner R: Nephrotoxicity - what do we know and what don't we know? Journal of pediatric hematology/oncology 33: 128-134, 2011.

2 Arany I, Clark JS, Ember I and Juncos LA: Epigenetic modifiers exert renal toxicity through induction of p66shc. Anticancer Res 31: 3267-3271, 2011.

3 Orth SR and Hallan SI: Smoking: a risk factor for progression of chronic kidney disease and for cardiovascular morbidity and mortality in renal patients - absence of evidence or evidence of absence? Clin J Am Soc Nephrol 3: 226-236, 2008.

4 Arany I, Grifoni S, Clark JS, Csongradi E, Maric C and Juncos LA: Chronic nicotine exposure exacerbates acute renal ischemic injury. Am J Physiol Renal Physiol 301: F125-133, 2011.

5 Jain $G$ and Jaimes EA: Nicotine signaling and progression of chronic kidney disease in smokers. Biochem Pharmacol 86: 1215-1223, 2013

6 Rezonzew G, Chumley P, Feng W, Hua P, Siegal G and Jaimes EA: Nicotine Exposure and the Progression of Chronic Kidney Disease: Role of the alpha7-nicotinic acetylcholine receptor. Am J Physiol Renal Physiol 303: F304-312, 2012.

7 Jaimes EA, Tian RX and Raij L: Nicotine: the link between cigarette smoking and the progression of renal injury? Am J Physiol Heart Circ Physiol 292: H76-82, 2007.

8 Arany I, Clark J, Reed DK and Juncos LA: Chronic nicotine exposure augments renal oxidative stress and injury through transcriptional activation of p66shc. Nephrol Dial Transplant 28: 1417-1425, 2013.

9 Rodrigo R and Bosco C: Oxidative stress and protective effects of polyphenols: comparative studies in human and rodent kidney. A review. Comp Biochem Physiol C Toxicol Pharmacol 142: 317-327, 2006.

10 Kurus M, Ugras M and Esrefoglu M: Effect of resveratrol on tubular damage and interstitial fibrosis in kidneys of rats exposed to cigarette smoke. Toxicology and industrial health 25 : 539-544, 2009.

11 Cheng L, Jin Z, Zhao R, Ren K, Deng C and Yu S: Resveratrol attenuates inflammation and oxidative stress induced by myocardial ischemia-reperfusion injury: role of Nrf2/ARE pathway. Int J Clin Exp Med 8: 10420-10428, 2015.
12 Hasko G and Pacher P: Endothelial Nrf2 activation: a new target for resveratrol? Am J Physiol Heart Circ Physiol 299: H10-12, 2010.

13 Arany I, Faisal A, Clark JS, Vera T, Baliga R and Nagamine Y: p66shc-mediated mitochondrial dysfunction in renal proximal tubule cells during oxidative injury. Am J Physiol Renal Physiol 298: F1214-1221, 2010.

14 Kim CS, Jung SB, Naqvi A, Hoffman TA, DeRicco J, Yamamori T, Cole MP, Jeon BH and Irani K: p53 impairs endotheliumdependent vasomotor function through transcriptional upregulation of p66shc. Circ Res 103: 1441-1450, 2008.

15 Alam J and Cook JL: Transcriptional regulation of the heme oxygenase-1 gene via the stress response element pathway. Curr Pharm Des 9: 2499-2511, 2003.

16 Arany I, Faisal A, Nagamine Y and Safirstein RL: p66shc inhibits pro-survival epidermal growth factor receptor/ERK signaling during severe oxidative stress in mouse renal proximal tubule cells. J Biol Chem 283: 6110-6117, 2008.

17 Arany I, Carter A, Hall S, Fulop T and Dixit M: Coenzyme Q10 protects renal proximal tubule cells against nicotine-induced apoptosis through induction of p66shc-dependent antioxidant responses. Apoptosis 22: 220-228, 2016.

18 Nadasi E, Clark JS, Szanyi I, Varjas T, Ember I, Baliga R and Arany I: Epigenetic modifiers exacerbate oxidative stress in renal proximal tubule cells. Anticancer Res 29: 2295-2299, 2009.

19 Niture SK, Kaspar JW, Shen J and Jaiswal AK: Nrf2 signaling and cell survival. Toxicol Appl Pharmacol 244: 37-42, 2010.

20 Petros WP, Younis IR, Ford JN and Weed SA: Effects of tobacco smoking and nicotine on cancer treatment. Pharmacotherapy 32: 920-931, 2012.

21 Gritz ER, Dresler C and Sarna L: Smoking, the missing drug interaction in clinical trials: ignoring the obvious. Cancer Epidemiol Biomarkers Prev 14: 2287-2293, 2005.

22 Chen CY, Jang JH, Li MH and Surh YJ: Resveratrol upregulates heme oxygenase-1 expression via activation of NF-E2-related factor 2 in PC12 cells. Biochem Biophys Res Commun 331: 993-1000, 2005.

23 Yang SK, Xiao L, Li J, Liu F and Sun L: Oxidative stress, a common molecular pathway for kidney disease: Role of the redox enzyme p66Shc. Ren Fail 36: 313-320, 2014.

24 Arany I, Faisal A, Clark JS, Vera T, Baliga R and Nagamine Y: p66SHC-mediated mitochondrial dysfunction in renal proximal tubule cells during oxidative injury. Am J Physiol Renal Physiol 298: F1214-1221, 2010

25 Miyazawa $M$ and Tsuji Y: Evidence for a novel antioxidant function and isoform-specific regulation of the human p66Shc gene. Mol Biol Cell 25: 2116-2127, 2014.

26 Fabbrocini G, Kisslinger A, Iannelli P, Vitale N, Procaccini C, Sparaneo G, Chieffi P, Ayala F, Mancini FP and Tramontano D: Resveratrol regulates p66Shc activation in HaCaT cells. Exp Dermatol 19: 895-903, 2010.

27 Conte A, Procaccini C, Iannelli P, Kisslinger A, De Amicis F, Pierantoni GM, Mancini FP, Matarese G and Tramontano D: Effects of Resveratrol on p66Shc phosphorylation in cultured prostate cells. Translational medicine @ UniSa 13: 47-58, 2015.

28 Arany I, Clark JS, Reed D, Szabo I, Ember I and Juncos LA: The Role of p66shc in Taxol- and Dichloroacetic Acid-dependent Renal Toxicity. Anticancer Res 33: 3119-3122, 2013.

Received May 31, 2017

Revised June 15, 2017

Accepted June 16, 2017 